ELECTRONIC RESEARCH ANNOUNCEMENTS OF THE AMERICAN MATHEMATICAL SOCIETY

Volume 9, Pages 10-18 (January 31, 2003)

S $1079-6762(03) 00107-0$

\title{
LEVI'S PARAMETRIX FOR SOME SUB-ELLIPTIC NON-DIVERGENCE FORM OPERATORS
}

\author{
ANDREA BONFIGLIOLI, ERMANNO LANCONELLI, AND FRANCESCO UGUZZONI
}

(Communicated by Michael Taylor)

\begin{abstract}
AвSTRACT. We construct the fundamental solutions for the sub-elliptic operators in non-divergence form $\sum_{i, j} a_{i, j}(x, t) X_{i} X_{j}-\partial_{t}$ and $\sum_{i, j} a_{i, j}(x) X_{i} X_{j}$, where the $X_{i}$ 's form a stratified system of Hörmander vector fields and $a_{i, j}$ are Hölder continuous functions belonging to a suitable class of ellipticity.
\end{abstract}

\section{MAin RESUlts}

Let $\mathbb{G}$ be a stratified group, i.e., let $\mathbb{G}$ be a connected and simply connected Lie group such that its Lie algebra $\mathfrak{g}$ admits the decomposition

$$
\mathfrak{g}=\mathfrak{G}_{1} \oplus \cdots \oplus \mathfrak{G}_{r}, \text { where }\left[\mathfrak{G}_{1}, \mathfrak{G}_{i}\right]=\mathfrak{G}_{i+1},\left[\mathfrak{G}_{1}, \mathfrak{G}_{r}\right]=\{0\}
$$

If $\left\{X_{1}, \ldots, X_{m}\right\}$ is a basis of $\mathfrak{G}_{1}$, we call the second order linear operator $\sum_{i=1}^{m} X_{i}^{2}$ a sub-Laplacian on $\mathbb{G}$. The study of general second order linear PDE's sum of squares of vector fields (started with Hörmander's paper [13]) has significantly developed after the works by Folland [11 and by Rothschild and Stein [23]; in the latter it has been shown that any general Hörmander operator can be locally approximated by a sub-Laplacian on a (free) stratified group $\mathbb{G}$. Since the appearance of this result, the study of stratified groups (also known as Carnot groups) has received great impulse from many authors and from different points of view. Second order linear and nonlinear PDE's of sub-elliptic type arise in various settings: geometric theory of several complex variables, curvature problems for CR-manifolds, subRiemannian geometry, diffusion processes, control theory, and human vision; see, e.g., [10, 15, 18, 21, 22, 24, 25]. The underlying algebraic structures of all these equations are stratified groups $\mathbb{G}$.

In this note, we present a result from a wider project aimed to apply analysis on stratified groups to the study of fully non-linear PDE's of sub-elliptic type arising in the geometric theory of several complex variables such as the Levi-curvature equation, which has achieved rising concern in the last few years (see [10, 14, 20. 24]). The core of this project is to study the fundamental solutions for the linear parabolic-type operators in non-divergence form on $\mathbb{G} \times \mathbb{R} \equiv \mathbb{R}^{N+1}$,

$$
\mathcal{H}=\sum_{i, j} a_{i, j}(x, t) X_{i} X_{j}-\partial_{t} \quad(x \in \mathbb{G}, t \in \mathbb{R}),
$$

where the $X_{i}$ 's are as above and $a_{i, j}$ are Hölder continuous functions belonging to

Received by the editors November 11, 2002 .

2000 Mathematics Subject Classification. Primary 35A08, 35H20, 43A80; Secondary 35A17, 35J70.

Key words and phrases. Non-divergence sub-elliptic operators, stratified groups, fundamental solutions, parametrix method.

Investigation supported by the University of Bologna Funds for selected research topics. 
a suitable class of ellipticity. Analogously, we study the fundamental solutions for the linear sub-elliptic non-divergence operators

$$
\mathcal{L}=\sum_{i, j} a_{i, j}(x) X_{i} X_{j}
$$

Operators such as $\mathcal{H}$ and $\mathcal{L}$ naturally intervene in the linearization of non-linear sub-elliptic equations. Then, the non-divergence form of $\mathcal{H}$ and $\mathcal{L}$ is a crucial requirement in view of our applications in non-linear PDE's analysis.

Several results concerning divergence form operators $-\sum_{i, j} X_{i}^{*}\left(a_{i, j} X_{j}\right)$ are present in the literature, both for linear and quasi-linear equations. Harnack's inequality, regularity results for solutions, existence and size estimates of the Green's function can be found, e.g., in the papers [6, 7, 9, 12, 19, 26]. On the contrary, at the authors' knowledge, very few papers are devoted to non-divergence form operators. We may just quote a work by Bramanti and Brandolini [5] and the recent paper by Capogna and Han [8, where a priori estimates in $L^{p}$ and Hölder spaces, respectively, are proved.

Here we discuss the existence and the well-behaved properties of the fundamental solutions for $\mathcal{H}$ and $\mathcal{L}$. We construct a fundamental solution $\Gamma$ for $\mathcal{H}$ by means of the well-known Levi's parametrix method, whereas a fundamental solution $\gamma$ for $\mathcal{L}$ is derived by a $t$-saturation argument. We explicitly remark that several new difficulties arise in the adaptation to our setting of the cited parametrix method. The major of these difficulties consists in obtaining suitable uniform estimates for the relevant frozen operators (see (1.4)). The derivation of these estimates is outlined in Section 2. Here we only point out that we make use of a lifting procedure to free stratified groups, and of an accurate analysis of the equivalence (via well-estimated automorphisms) of all sub-Laplacians on such free stratified groups. Moreover, the lack of knowledge of an explicit expression for the parametrix clearly makes Levi's method more involved than in the classical context.

By integrating $\Gamma$ over the time variable $t$, we are then able to construct also a local fundamental solution $\gamma$ for $\mathcal{L}$. This can be done provided suitable longtime estimates of $\Gamma$ are established. We stress that, whereas optimal small-time estimates of $\Gamma$ and of its derivatives can be directly obtained from the construction of $\Gamma$, a more delicate matter is to show long-time estimates. We are able to obtain the latter up to a suitable modification of the coefficients $a_{i, j}$ outside a compact set (see Lemma 3.3 below).

We hereafter announce our main results (all the notations are explained below). For the complete proofs we refer to 2$]$.

Theorem 1.1. Suppose the coefficients $a_{i, j}$ of $\mathcal{H}$ belong to the ellipticity class $\mathcal{M}_{\Lambda}$ and to the Hölder space $\Gamma^{\alpha}\left(\mathbb{R}^{N+1}\right)$. Then there exists a fundamental solution $\Gamma$ for $\mathcal{H}$, with the properties listed below.

(i) $\Gamma$ is a continuous function away from the diagonal of $\mathbb{R}^{N+1} \times \mathbb{R}^{N+1}$. Moreover, for every fixed $\zeta \in \mathbb{R}^{N+1}, \Gamma(\cdot ; \zeta) \in \Gamma_{\text {loc }}^{2+\alpha}\left(\mathbb{R}^{N+1} \backslash\{\zeta\}\right)$ and we have $\mathcal{H}(\Gamma(\cdot ; \zeta))=0$ in $\mathbb{R}^{N+1} \backslash\{\zeta\}$.

(ii) $\Gamma(x, t ; \xi, \tau)=0$ for $t \leq \tau$. Moreover, there exists a positive constant $M$ and, for every $T>0$, there exists a positive constant $c(T)$ such that, for $0<t-\tau \leq T$, the following estimates hold:

$$
0 \leq \Gamma(x, t ; \xi, \tau) \leq c(T)(t-\tau)^{-Q / 2} \exp \left(-\frac{d^{2}(x, \xi)}{M(t-\tau)}\right),
$$




$$
\begin{aligned}
&\left|X_{i}(\Gamma(\cdot ; \xi, \tau))(x, t)\right| \leq c(T)(t-\tau)^{-(Q+1) / 2} \exp \left(-\frac{d^{2}(x, \xi)}{M(t-\tau)}\right), \\
&\left|X_{i} X_{j}(\Gamma(\cdot ; \xi, \tau))(x, t)\right|,\left|\partial_{t}(\Gamma(\cdot ; \xi, \tau))(x, t)\right| \\
& \leq c(T)(t-\tau)^{-(Q+2) / 2} \exp \left(-\frac{d^{2}(x, \xi)}{M(t-\tau)}\right) .
\end{aligned}
$$

(iii) For every $\psi \in C_{0}^{\infty}\left(\mathbb{R}^{N+1}\right)$, the convolution $w(z)=\int_{\mathbb{R}^{N+1}} \Gamma(z ; \zeta) \psi(\zeta) \mathrm{d} \zeta$ belongs to the class $\Gamma_{\text {loc }}^{2+\alpha}\left(\mathbb{R}^{N+1}\right)$ and we have $\mathcal{H} w=-\psi$ in $\mathbb{R}^{N+1}$.

(iv) Let $\mu \geq 0$ and $T_{2}>T_{1}$ be such that $\left(T_{2}-T_{1}\right) \mu$ is small enough. Then, for every $f \in \Gamma^{\beta}\left(\mathbb{R}^{N} \times\left[T_{1}, T_{2}\right]\right.$ ) (where $0<\beta \leq \alpha$ ) and $g \in C\left(\mathbb{R}^{N}\right)$ satisfying the growth condition $|f(x, t)|,|g(x)| \leq c \exp \left(\mu d^{2}(x)\right)$ for some constant $c>0$, the function (defined for $x \in \mathbb{R}^{N}, t \in\left(T_{1}, T_{2}\right]$ )

$$
u(x, t)=\int_{\mathbb{R}^{N}} \Gamma\left(x, t ; \xi, T_{1}\right) g(\xi) \mathrm{d} \xi+\int_{\mathbb{R}^{N} \times\left[T_{1}, t\right]} \Gamma(x, t ; \xi, \tau) f(\xi, \tau) \mathrm{d} \xi \mathrm{d} \tau,
$$

belongs to the class $\Gamma_{\text {loc }}^{2+\beta}\left(\mathbb{R}^{N} \times\left(T_{1}, T_{2}\right)\right) \cap C\left(\mathbb{R}^{N} \times\left[T_{1}, T_{2}\right]\right)$. Moreover, $u$ is a solution to the Cauchy problem $\mathcal{H} u=-f$ in $\mathbb{R}^{N} \times\left(T_{1}, T_{2}\right), u\left(\cdot, T_{1}\right)=g$.

Theorem 1.2. Suppose that the dimension $m$ of $\mathfrak{G}_{1}$ is greater than two. Suppose the coefficients $a_{i, j}$ of $\mathcal{L}$ are in the Hölder space $\Gamma^{\alpha}\left(\mathbb{R}^{N}\right)$ and in the ellipticity class $\mathcal{M}_{\Lambda}$. For every fixed bounded open set $\Omega \subset \mathbb{R}^{N}$, there exists a fundamental solution $\gamma$ for $\mathcal{L}$ in $\Omega$, with the properties listed below.

(i) $\gamma$ is a continuous function away from the diagonal of $\mathbb{R}^{N} \times \mathbb{R}^{N}$. Moreover, for every fixed $\xi \in \mathbb{R}^{N}, \gamma(\cdot, \xi) \in \Gamma_{\text {loc }}^{2+\alpha}\left(\mathbb{R}^{N} \backslash\{\xi\}\right)$ and we have $\mathcal{L}(\gamma(\cdot, \xi))=0$ in $\Omega \backslash\{\xi\}$.

(ii) For every compact set $K \Subset \mathbb{R}^{N}$, there exists a positive constant $c$ such that

$$
0 \leq \gamma(x, \xi) \leq c\left(1+d(x, \xi)^{2-Q}\right), \quad \xi \in K, x \in \mathbb{R}^{N} .
$$

(iii) For every $\psi \in C_{0}^{\infty}\left(\mathbb{R}^{N}\right)$, the convolution $w(x)=\int_{\mathbb{R}^{N}} \gamma(x ; \xi) \psi(\xi) \mathrm{d} \xi$ belongs to the class $\Gamma_{\text {loc }}^{2+\alpha}\left(\mathbb{R}^{N}\right)$ and we have $\mathcal{L} w=-\psi$ in $\Omega$.

In the above theorems, we have denoted by $Q=\sum_{j=1}^{r} j \operatorname{dim}\left(\mathfrak{G}_{j}\right)$ the homogeneous dimension of $\mathbb{G}$, by $d$ a fixed homogeneous norm on $\mathbb{G}$ and we have set $d(x, y)=d\left(y^{-1} \circ x\right)$. Moreover, given $\Lambda>1$, we have denoted by $\mathcal{M}_{\Lambda}$ the ellipticity class of the $m \times m$ symmetric matrices $A$ such that $\Lambda^{-1}|\xi|^{2} \leq\langle A \xi, \xi\rangle \leq \Lambda|\xi|^{2}$, for every $\xi \in \mathbb{R}^{m}$. Finally we have denoted by $\Gamma^{\beta}, \Gamma^{2+\beta}$ the appropriate sub-elliptic Hölder spaces.

As is well known, Levi's parametrix method requires the knowledge of several good properties of the frozen constant coefficient parabolic-type operators

$$
\mathcal{H}_{A}=\mathcal{L}_{A}-\partial_{t}=\sum_{i, j=1}^{m} a_{i, j} X_{i} X_{j}-\partial_{t},
$$

where $A=\left(a_{i, j}\right)_{i, j}$ is a fixed matrix in the ellipticity class $\mathcal{M}_{\Lambda}$. For instance, a crucial role is played by the following uniform Gaussian estimates for the fundamental solutions $\Gamma_{A}$ of $\mathcal{H}_{A}$ :

$$
\begin{aligned}
\mid X_{i_{1}} \cdots X_{i_{p}}\left(\partial_{t}\right)^{q} & \Gamma_{A}(x, t)-X_{i_{1}} \cdots X_{i_{p}}\left(\partial_{t}\right)^{q} \Gamma_{B}(x, t) \mid \\
& \leq c_{\Lambda, p, q}\|A-B\|^{1 / r} t^{-(Q+p+2 q) / 2} \exp \left(-\frac{d^{2}(x)}{c_{\Lambda} t}\right),
\end{aligned}
$$


for every $A, B \in \mathcal{M}_{\Lambda}$ (here, $\|A\|$ denotes the matrix norm $\max _{|\xi|=1}|A \xi|$ ). Gaussian estimates, but not uniform, for heat kernels on Lie groups were proved by Jerison and Sànchez-Calle [16], by Kusuoka and Stroock [17] and by Varopoulos, SaloffCoste and Coulhon [25]. Uniform estimates, but not Gaussian, for families of Hörmander operators generalizing (1.1), were proved by Rothschild and Stein [23] and by Bramanti and Brandolini [5].

Since the derivation of uniform and Gaussian estimates is a non-trivial task and is a key point in proving the main results we announce here, in Section 2 we briefly recall how (1.4) can be established by a direct approach. In Section 3 we briefly outline the proof of Theorem 1.1 describing our adaptation of the parametrix method and how uniform estimates naturally intervene. Finally, we sketch how long-time estimates are used in order to prove Theorem 1.2 employing both a $t$-saturation and an approximation argument.

\section{BACKGROUND MATERIAL}

The classical Levi's parametrix method (i.e., if the underlying stratified group is the usual Euclidean space $\left.\left(\mathbb{R}^{N},+\right)\right)$ exploits at various levels the explicit knowledge of the fundamental solution for the strictly parabolic constant coefficient operator $\sum_{i, j=1}^{N} a_{i, j} \partial_{i} \partial_{j}-\partial_{t}\left(A=\left(a_{i, j}\right)_{i, j}\right.$ being a positive-definite matrix $)$. Obviously, this fundamental solution is given by the composition of the fundamental solution of the classical heat operator $\sum_{i=1}^{N}\left(\partial_{i}\right)^{2}-\partial_{t}$ on $\mathbb{R}^{N+1}$ with a linear change of coordinates related to the matrix $A^{-1 / 2}$.

A naïve idea in studying the fundamental solutions for (1.3) and in approaching (1.4) is to ask if something similar may occur in the case of general stratified groups $\mathbb{G}$. Namely, we ask if all sub-Laplacians on $\mathbb{G}$ can be put (via a diffeomorphism) into a fixed canonical form. To this end, if $A=\left(a_{i, j}\right)_{i, j \leq m}$ is a positive-definite symmetric matrix, we first remark that $\mathcal{L}_{A}=\sum_{i, j=1}^{m} a_{i, j} X_{i} X_{j}$ may be rewritten as $\sum_{i=1}^{m} Y_{i}^{2}$, where $Y_{i}=\sum_{j=1}^{m}\left(A^{1 / 2}\right)_{i, j} X_{j}$. As a consequence, it is natural to ask if there exists a diffeomorphism $T_{A}: \mathbb{G} \rightarrow \mathbb{G}$ such that (in the new coordinate system defined by $T_{A}$ ) the vector field $Y_{i}$ is turned into the (fixed) left-invariant vector field $Z_{i}$ agreeing at the origin with $\partial_{i}$. In this way, if we set $\Delta_{\mathbb{G}}=\sum_{i=1}^{m} Z_{i}^{2}$ (we call $\Delta_{\mathbb{G}}$ the canonical sub-Laplacian on $\mathbb{G}$ ), the sub-Laplacian $\sum_{i=1}^{m} Y_{i}^{2}$ is turned into $\Delta_{\mathbb{G}}$, i.e.,

$$
\mathcal{L}_{A}\left(u \circ T_{A}\right)=\left(\Delta_{\mathbb{G}} u\right) \circ T_{A}, \quad \text { for every } u \in C^{\infty}(\mathbb{G}) .
$$

In the classical case when $X_{i}=\partial / \partial x_{i}$, this problem always has a solution. On the contrary, counterexamples can be given showing that $T_{A}$ may not exist for general stratified groups $\mathbb{G}$ and, when it exists, it may be non-linear (see [4]). Broadly speaking, the problem relies on the commutativity properties of the $X_{i}$ 's: if the linear dependence relations among commutators up to order $r$ of $X_{1}, \ldots, X_{m}$ are the least possible, then the above problem does have a solution. More explicitly, if $\mathbb{G}$ is a free stratified group (i.e., its Lie algebra is isomorphic to a free nilpotent Lie algebra) then there exists an automorphism $T_{A}$ of the group $\mathbb{G}$ satisfying (2.1).

As a straightforward consequence, when $\mathbb{G}$ is free, we are able to obtain the fundamental solution $\Gamma_{A}$ for $\mathcal{H}_{A}=\mathcal{L}_{A}-\partial_{t}$ simply as the composition of $T_{A}$ with the fundamental solution $\Gamma_{\mathbb{G}}$ for the fixed canonical heat operator $\Delta_{\mathbb{G}}-\partial_{t}$. Indeed, if $\mathbb{G}$ is free, it turns out that

$$
\Gamma_{A}(x, t ; \xi, \tau)=\left|\operatorname{det} \mathcal{J}_{T_{A}}(x)\right| \Gamma_{\mathbb{G}}\left(T_{A}(x), t ; T_{A}(\xi), \tau\right), \quad x, \xi \in \mathbb{R}^{N}, t, \tau \in \mathbb{R}
$$


$\left(\mathcal{J}_{T_{A}}\right.$ denotes the Jacobian matrix of $\left.T_{A}\right)$. Thanks to this somewhat explicit representation of the fundamental solution for $\mathcal{H}_{A}$, the next step in order to obtain the uniform estimates in (1.4) is to establish ad hoc uniform estimates for $T_{A}$. To this end it can be proved that $\left|\operatorname{det} \mathcal{J}_{T_{A}}(x)\right|$ turns out to be a uniformly bounded constant and that $d\left(T_{A}(x), T_{B}(x)\right) \leq c_{\Lambda}\|A-B\|^{1 / r} d(x)$ for every $A, B \in \mathcal{M}_{\Lambda}$ and $x \in \mathbb{G}$. Consequently (see [1]), when $\mathbb{G}$ is free, the uniform Gaussian estimates (1.4) follow from (2.2) and from the following Gaussian estimates of the (fixed) fundamental solution $\Gamma_{\mathbb{G}}$ :

$$
\left|X_{i_{1}} \cdots X_{i_{p}}\left(\partial_{t}\right)^{q} \Gamma_{\mathbb{G}}(x, t)\right| \leq c_{\mathbb{G}} t^{-(Q+p+2 q) / 2} \exp \left(-\frac{d^{2}(x)}{c_{\mathbb{G}} t}\right) .
$$

In order to handle the case of an arbitrary stratified group $\mathbb{G}$, our main tool is to lift $\mathbb{G}$ to a free stratified group $\widetilde{\mathbb{G}}$ in such a way that $\Delta_{\mathbb{G}}$ is lifted to $\Delta_{\widetilde{\mathbb{G}}}$. The lifting technique introduced by Rothschild and Stein in [23] together with some further remarks on the homogeneity properties of stratified groups, allows us to prove the following lifting result (see also [3]): If $\mathbb{G}$ is an $N$-dimensional stratified group then there exists an $H$-dimensional free stratified group $\widetilde{\mathbb{G}}$ (with $H \geq N$ ) such that, denoting by $\pi: \mathbb{R}^{H} \rightarrow \mathbb{R}^{N}$ the projection on the first $N$ coordinates, for every $u \in C^{\infty}(\mathbb{G})$, we have $\widetilde{Z}_{i}(u \circ \pi)=\left(Z_{i} u\right) \circ \pi$, where $\sum_{i=1}^{m} Z_{i}^{2}$ and $\sum_{i=1}^{m} \widetilde{Z}_{i}^{2}$ are the canonical sub-Laplacians $\Delta_{\mathbb{G}}$ and $\Delta_{\widetilde{G}}$, respectively. Since $\mathfrak{G}_{1}=\operatorname{span}\left\{Z_{1}, \ldots, Z_{m}\right\}$, the lifting result leads to a correspondence between the operators $\mathcal{H}_{A}=\sum_{i, j=1}^{m} a_{i, j} X_{i} X_{j}-\partial_{t}$ on $\mathbb{G} \times \mathbb{R}$ and $\widetilde{\mathcal{H}}_{A}=\sum_{i, j=1}^{m} a_{i, j} \widetilde{X}_{i} \widetilde{X}_{j}-\partial_{t}$ on $\widetilde{\mathbb{G}} \times \mathbb{R}$.

The lifting result also allows us to establish a natural relation between the fundamental solution $\Gamma_{A}$ for $\mathcal{H}_{A}$ and $\widetilde{\Gamma}_{A}$ for $\widetilde{\mathcal{H}}_{A}$. Indeed, we have

$$
\Gamma_{A}(x, t)=\int_{\mathbb{R}^{H-N}} \widetilde{\Gamma}_{A}((x, \widehat{x}), t) \mathrm{d} \widehat{x}, \quad \text { for every } x \in \mathbb{G}, t \in \mathbb{R},
$$

where $(x, \widehat{x})$ denotes the point of $\mathbb{R}^{N} \times \mathbb{R}^{H-N}$. This fact, together with the established uniform estimates for free groups, allows us to prove (1.4) in the general case. Indeed, by means of the integral representation in (2.3), it is possible to transfer the uniform estimates for $\left\{\widetilde{\Gamma}_{A}\right\}$ to the uniform estimates for $\left\{\Gamma_{A}\right\}$.

\section{LEVI'S PARAMETRIX METHOD}

We first fix some notation. The point of $\mathbb{R}^{N+1}$ will be denoted by $z=(x, t)$ $\left(x \in \mathbb{R}^{N}, t \in \mathbb{R}\right)$ and analogously $\zeta=(\xi, \tau)$. The coefficients $a_{i, j}$ of the operator $\mathcal{H}$ in (1.1) will be assumed to satisfy

$$
\left(a_{i, j}(x, t)\right)_{i, j} \in \mathcal{M}_{\Lambda}, \quad\left|a_{i, j}(x, t)-a_{i, j}\left(x^{\prime}, t^{\prime}\right)\right| \leq L\left(d\left(x, x^{\prime}\right)^{\alpha}+\left|t-t^{\prime}\right|^{\alpha / 2}\right) .
$$

All constants will be meant to depend on $\Lambda, L, \alpha$. Following (1.3), we set for brevity $\mathcal{H}_{\zeta_{0}}:=\mathcal{H}_{A\left(\zeta_{0}\right)}$ (and analogously, $\Gamma_{\zeta_{0}}:=\Gamma_{A\left(\zeta_{0}\right)}$ ). The uniform Gaussian estimates discussed in Section 2 (see, e.g., (1.4)) allow us to prove the following estimates of the fundamental solutions $\Gamma_{\zeta_{0}}$ for the frozen operators $\mathcal{H}_{\zeta_{0}}$ (uniform in $\zeta_{0}$ ):

$$
\begin{aligned}
& c^{-1} \mathbf{E}\left(x, c^{-1} t\right) \leq \Gamma_{\zeta_{0}}(x, t) \leq c \mathbf{E}(x, c t) \\
& \left|X_{i_{1}} \cdots X_{i_{p}}\left(\partial_{t}\right)^{q} \Gamma_{\zeta_{0}}(x, t)\right| \leq c_{p, q} t^{-(p+2 q) / 2} \mathbf{E}(x, c t) \\
& \left|X_{i_{1}} \cdots X_{i_{p}}\left(\partial_{t}\right)^{q} \Gamma_{\zeta_{0}}(x, t)-X_{i_{1}} \cdots X_{i_{p}}\left(\partial_{t}\right)^{q} \Gamma_{\zeta_{1}}(x, t)\right| \\
& \quad \leq \mathbf{c}_{p, q}\left(d\left(\xi_{0}, \xi_{1}\right)^{\frac{\alpha}{r}}+\mid \tau_{0}-\tau_{1}{ }^{\frac{\alpha}{2 r}}\right) t^{-(p+2 q) / 2} \mathbf{E}(x, c t),
\end{aligned}
$$


where $\mathbf{E}(x, t)=t^{-Q / 2} \exp \left(-d(x)^{2} / t\right)$. We remark that the parametrix method outlined below is classical, but several technical complications arise in our setting. We set $($ for $z \neq \zeta$ )

$$
Z_{1}(z ; \zeta)=\mathcal{H}\left(z \mapsto \Gamma_{\zeta}\left(\xi^{-1} \circ x, t-\tau\right)\right)(z)
$$

Since $\mathcal{H}=\mathcal{L}-\mathcal{L}_{\zeta}+\mathcal{H}_{\zeta}$, we have

$$
Z_{1}(z ; \zeta)=\sum_{i, j=1}^{m}\left(a_{i, j}(z)-a_{i, j}(\zeta)\right) X_{i} X_{j} \Gamma_{\zeta}\left(\xi^{-1} \circ x, t-\tau\right),
$$

whence (3.1) gives $\left|Z_{1}(z ; \zeta)\right| \leq c(t-\tau)^{\frac{\alpha}{2}-1} \Gamma_{\zeta_{0}}\left(\xi^{-1} \circ x, c(t-\tau)\right)$. If we inductively define

$$
Z_{j+1}(z ; \zeta)=\int_{\mathbb{R}^{N} \times[\tau, t]} Z_{1}(z ; \eta) Z_{j}(\eta ; \zeta) \mathrm{d} \eta \quad(t>\tau)
$$

the following estimate holds (for suitable constants $c_{1}, c_{2}, b_{j}$ )

$$
\left|Z_{j}(z ; \zeta)\right| \leq c_{1}^{j} b_{j}(\alpha)(t-\tau)^{-1+j \alpha / 2} \Gamma_{\zeta_{0}}\left(\xi^{-1} \circ x, c_{2}(t-\tau)\right) .
$$

By means of (3.1), it can then be proved that the series $\Phi(z ; \zeta)=\sum_{j=1}^{\infty} Z_{j}(z ; \zeta)$ totally converges on a suitable domain and satisfies the estimate (here $T>0$ and $c(T)>0$ is a constant)

$$
|\Phi(z ; \zeta)| \leq c(T)(t-\tau)^{\frac{\alpha}{2}-1} \mathbf{E}\left(\xi^{-1} \circ x, c(t-\tau)\right), \quad 0<t-\tau \leq T .
$$

It is easy to see that (for $t>\tau) \Phi(z ; \zeta)=Z_{1}(z ; \zeta)+\int_{\mathbb{R}^{N} \times[\tau, t]} Z_{1}(z ; \eta) \Phi(\eta ; \zeta) \mathrm{d} \eta$. A crucial tool in the adaptation of the parametrix method is played by the following non-trivial regularity properties of $\Phi: \Phi(\cdot ; \zeta)$ and $\Phi(z ; \cdot)$ are continuous functions and (for $0<t-\tau \leq T$ )

$$
\begin{aligned}
& \left|\Phi(x, t ; \xi, \tau)-\Phi\left(x^{\prime}, t ; \xi, \tau\right)\right| \\
& \quad \leq c(T) d\left(x, x^{\prime}\right)^{\frac{\alpha}{2}}(t-\tau)^{\frac{\alpha}{4}-1}\left(\mathbf{E}\left(\xi^{-1} \circ x, c(t-\tau)\right)+\mathbf{E}\left(\xi^{-1} \circ x^{\prime}, c(t-\tau)\right)\right) .
\end{aligned}
$$

We now set, for every $z, \zeta \in \mathbb{R}^{N+1}$ with $t>\tau$,

$$
J(z ; \zeta)=\int_{\mathbb{R}^{N} \times[\tau, t]} \Gamma_{\eta}\left(z ; z^{\prime}\right) \Phi\left(z^{\prime} ; \zeta\right) \mathrm{d} z^{\prime}, \quad \Gamma(z ; \zeta)=J(z ; \zeta)+\Gamma_{\zeta}(z ; \zeta),
$$

and extend $\Gamma(z ; \zeta)$ to be zero for $t \leq \tau$. The good property (3.2) of $\Phi$ and the Gaussian estimates (3.1) ensure that $\Gamma$ is well posed. Exploiting again the estimates (3.1) and (3.2), it is not difficult to show that $\Gamma$ is continuous away from the diagonal of $\mathbb{R}^{N+1} \times \mathbb{R}^{N+1}$ and satisfies

$$
|\Gamma(z ; \zeta)| \leq c(T) \mathbf{E}\left(\xi^{-1} \circ x, c(t-\tau)\right) \quad(0<t-\tau \leq T) .
$$

We now show that $\Gamma$ is the fundamental solution for $\mathcal{H}$. The first step is to prove that $\mathcal{H}(\Gamma(\cdot ; \zeta))=0$ in $\mathbb{R}^{N+1} \backslash\{\zeta\}$. We start by giving the definition of a regularity class of functions that will be useful in the sequel: given an open set $\Omega \subseteq \mathbb{R}^{N+1}$, we shall denote by $\mathfrak{C}^{2}(\Omega)$ the class of functions $u(x, t)$ defined on $\Omega$ which are continuous in $\Omega$ with respect to the pair $(x, t)$ and such that $u(\cdot, t)$ has continuous Lie-derivatives up to second order along the vector fields $X_{1}, \ldots, X_{m}$ (with respect to $x$, for every fixed $t$ ) and $u(x, \cdot)$ has continuous derivative (with respect to $t$, for every fixed $x$ ), in their respective domains of definition. The class $\mathfrak{C}^{2}(\Omega)$ is larger than the natural space $\Gamma^{2}(\Omega)$ (of continuous functions $u$ with continuous derivatives $\left.X_{i} u, X_{i} X_{j} u, \partial_{t} u\right)$; nevertheless, we are able to prove a weak maximum principle for solutions to $\mathcal{H} u \geq 0$ in the class $\mathfrak{C}^{2}(\Omega)$, as stated below. 
Proposition 3.1. Let $\Omega$ be a bounded open subset of $\mathbb{R}^{N+1}$ and $t_{0} \in \mathbb{R}$; if $u \in$ $\mathfrak{C}^{2}(\Omega), \mathcal{H} u \geq 0$ in $\Omega \cap\left\{t<t_{0}\right\}$ and $\lim \sup u \leq 0$ in $\partial \Omega \cap\left\{t \leq t_{0}\right\}$, then $u \leq 0$ in $\Omega \cap\left\{t<t_{0}\right\}$.

With the above notation we are able to prove the following result.

Proposition 3.2. For every fixed $\zeta \in \mathbb{R}^{N+1}$, we have $\Gamma(\cdot ; \zeta) \in \mathfrak{C}^{2}\left(\mathbb{R}^{N+1} \backslash\{\zeta\}\right)$ and $\mathcal{H}(\Gamma(\cdot ; \zeta))=0$ in $\mathbb{R}^{N+1} \backslash\{\zeta\}$. Moreover, the estimates in (1.2) hold.

The main tool in the proof of the above proposition is the representation of the Lie-derivatives of the convolution (3.4) defining $J$ as principal-value (singular) integrals. Indeed, we have

$$
\begin{aligned}
& X_{i} X_{j}(J(\cdot ; \zeta))(z)=\lim _{\varepsilon \rightarrow 0^{+}} \int_{\mathbb{R}^{N} \times[\tau, t-\varepsilon]} X_{i} X_{j} \Gamma_{z^{\prime}}\left(x^{\prime-1} \circ x, t-t^{\prime}\right) \Phi\left(z^{\prime} ; \zeta\right) \mathrm{d} z^{\prime}, \\
& \partial_{t}(J(\cdot ; \zeta))(z)=\Phi(z ; \zeta)+\lim _{\varepsilon \rightarrow 0^{+}} \int_{\mathbb{R}^{N} \times[\tau, t-\varepsilon]} \partial_{t} \Gamma_{z^{\prime}}\left(x^{\prime-1} \circ x, t-t^{\prime}\right) \Phi\left(z^{\prime} ; \zeta\right) \mathrm{d} z^{\prime} .
\end{aligned}
$$

The proof of this fact is not straightforward; one of the main ingredients is the Hölder estimate of $\Phi$ in (3.3). It is now easy to see that

$$
\begin{aligned}
\mathcal{H}(\Gamma(\cdot ; \zeta))(z)= & \mathcal{H}\left(\Gamma_{\zeta}(\cdot ; \zeta)\right)(z) \\
& +\lim _{\varepsilon \rightarrow 0^{+}} \int_{\mathbb{R}^{N} \times[\tau, t-\varepsilon]} \mathcal{H}\left(\Gamma_{\eta}\left(\cdot ; z^{\prime}\right)\right)(z) \Phi\left(z^{\prime} ; \zeta\right) \mathrm{d} z^{\prime}-\Phi(z ; \zeta) \\
= & Z_{1}(z ; \zeta)+\int_{\mathbb{R}^{N} \times[\tau, t]} Z_{1}(z ; \eta) \Phi(\eta ; \zeta) \mathrm{d} \eta-\Phi(z ; \zeta)=0 .
\end{aligned}
$$

We avoid giving details here on the results (iii) and (iv) of Theorem 1.1 concerning the Cauchy problem related to $\mathcal{H}$.

We now turn to the study of the operator $\mathcal{L}=\sum_{i, j} a_{i, j}(x) X_{i} X_{j}$. Starting from Theorem 1.1, one can construct local fundamental solutions for $\mathcal{L}$, by integrating $\Gamma$ over the time variable $t$, provided suitable long-time estimates of $\Gamma$ can be established. We are able to find the needed long-time estimates if the dimension $m$ of the first layer $\mathfrak{G}_{1}$ of the stratification of $\mathfrak{g}$ is strictly greater than two. Indeed, we can prove the following technical lemma.

Lemma 3.3. Let $\Omega$ be a bounded domain of $\mathbb{R}^{N+1}$. Up to a modification of the coefficients $a_{i, j}(x, t)$ of $\mathcal{H}$ outside $\Omega, \Gamma$ satisfies the following estimate: for every compact set $K \Subset \mathbb{R}^{m}$, there exist positive constants $c, M$ such that

$$
\Gamma(x, t ; \xi, \tau) \leq c(M+t-\tau)^{-m / 2} \exp \left(-\frac{\Lambda\left|x^{(1)}-\xi^{(1)}\right|^{2}}{M+t-\tau}\right)
$$

(for $t>\tau+1$ and $\xi^{(1)} \in K$ ). We have denoted by $x^{(1)}$ the vector of the first $m$ coordinates of $x$.

The proof of Lemma 3.3 relies on the weak maximum principle for $\mathcal{H}$ in the class $\mathfrak{C}^{2}$ (see Proposition 3.1) and on a direct comparison argument. The above long-time estimate of $\Gamma$ is certainly not optimal, but it is indeed sufficient to ensure the convergence of the integral $\int_{\mathbb{R}} \Gamma(x, t ; \xi, 0) \mathrm{d} t$ (for $x \neq \xi$ ) if $m>2$. This allows us to obtain the result in Theorem 1.2 by arguing as sketched below.

We fix a cut-off function $\varphi \in C_{0}^{\infty}\left(\mathbb{R}^{N}\right)$ such that $0 \leq \varphi \leq 1$ and $\varphi \equiv 1$ in $\Omega$. We next define a suitable "GG-regularization" $A^{\varepsilon}=\left(a_{i, j}^{\varepsilon}\right)_{i, j}$ of the coefficients $a_{i, j}$ of $\mathcal{L}$, and we set

$$
\widetilde{A}^{\varepsilon}(x)=\varphi(x) A^{\varepsilon}(x)+(1-\varphi(x))(4 \Lambda)^{-1} \mathbb{I}_{m}
$$


(where $\mathbb{I}_{m}$ denotes the $m \times m$ identity matrix) and

$$
\widetilde{\mathcal{H}}^{\varepsilon}=\widetilde{\mathcal{L}}^{\varepsilon}-\partial_{t}=\sum_{i, j=1}^{m} \widetilde{a}_{i, j}^{\varepsilon}(x) X_{i} X_{j}-\partial_{t} .
$$

The coefficients have been suitably modified outside $\Omega$ so that we can apply Lemma 3.3 to ensure that the integral

$$
\widetilde{\gamma}^{\varepsilon}(x, \xi)=\int_{0}^{\infty} \widetilde{\Gamma}^{\varepsilon}(x, t ; \xi, 0) \mathrm{d} t, \quad x \neq \xi \in \mathbb{R}^{N},
$$

is convergent. Moreover, since the approximating operator $\widetilde{\mathcal{L}}^{\varepsilon}$ has smooth coefficients, one can prove that $\widetilde{\gamma}^{\varepsilon}$ is a fundamental solution for $\widetilde{\mathcal{L}}^{\varepsilon}$ by making use of the well-posedness of the adjoint operators $\left(\widetilde{\mathcal{H}}^{\varepsilon}\right)^{*}$ and $\left(\widetilde{\mathcal{L}}^{\varepsilon}\right)^{*}$. Indeed, for every test function $\psi \in C_{0}^{\infty}\left(\mathbb{R}^{N}\right.$ ), setting $\psi_{n}(x, t)=\psi(x) \theta(|t| / n)$ (where $\theta$ is a smooth function defined on $\mathbb{R}$, such that $\theta(s)=1$ for $s \leq 1$, and $\theta(s)=0$ for $s \geq 1$ ) we obtain

$$
\begin{aligned}
-\psi(\xi) & =-\psi_{n}(\xi, 0)=\int_{\mathbb{R}^{N+1}} \widetilde{\Gamma}^{\varepsilon}(x, t ; \xi, 0)\left(\widetilde{\mathcal{H}}^{\varepsilon}\right)^{*} \psi_{n}(x, t) \mathrm{d} \xi \mathrm{d} t \\
& \stackrel{n \rightarrow \infty}{\longrightarrow} \int_{\mathbb{R}^{N+1}} \widetilde{\Gamma}^{\varepsilon}(x, t ; \xi, 0)\left(\widetilde{\mathcal{L}}^{\varepsilon}\right)^{*} \psi(x) \mathrm{d} x \mathrm{~d} t=\int_{\mathbb{R}^{N}} \widetilde{\gamma}^{\varepsilon}(x, \xi)\left(\widetilde{\mathcal{L}}^{\varepsilon}\right)^{*} \psi(x) \mathrm{d} x .
\end{aligned}
$$

We finally find a fundamental solution $\widetilde{\gamma}$ of

$$
\widetilde{\mathcal{L}}=\sum_{i, j=1}^{m} \widetilde{a}_{i, j}(x) X_{i} X_{j}
$$

(where $\widetilde{A}=\varphi A+(1-\varphi)(4 \Lambda)^{-1} \mathbb{I}_{m}$ ), by an approximation argument, by letting $\varepsilon$ tend to zero in $\widetilde{\gamma}^{\varepsilon}$. This approximation argument is not trivial and it requires suitable Schauder-type a priori estimates and a careful study of the $\varepsilon$-dependence in the construction of $\widetilde{\Gamma}^{\varepsilon}$. We omit details here. Finally, observing that $\mathcal{L}=\widetilde{\mathcal{L}}$ in $\Omega$, we are then able to prove Theorem 1.2 .

\section{REFERENCES}

[1] Bonfiglioli, A., Lanconelli, E., Uguzzoni, F., Uniform Gaussian estimates of the fundamental solutions for heat operators on Carnot groups, Adv. Differential Equations 7 (2002), 1153-1192.

[2] Bonfiglioli, A., Lanconelli, E., UGuzzoni, F., Fundamental solutions for nondivergence form operators on stratified groups, preprint.

[3] Bonfiglioli, A., Uguzzoni, F., A note on lifting of Carnot groups, preprint.

[4] Bonfiglioli, A., Uguzzoni, F., Families of diffeomorphic sub-Laplacians and free Carnot groups, to appear in Forum Math.

[5] Bramanti, M., Brandolini, L., $L^{p}$ estimates for nonvariational hypoelliptic operators with VMO coefficients, Trans. Amer. Math. Soc. 352 (2000), no. 2, 781-822. MR 2000c:35026

[6] Capogna, L., Regularity for quasilinear equations and 1-quasiconformal maps in Carnot groups, Math. Ann. 313 (1999), no. 2, 263-295. MR 2000a:35027

[7] Capogna, L., Danielli, D., Garofalo, N., Capacitary estimates and the local behavior of solutions of nonlinear subelliptic equations, Amer. J. Math. 118 (1996), no. 6, 1153-1196. MR 97k:35033

[8] Capogna, L., Han, Q., Pointwise Schauder estimates for second order linear equations in Carnot groups, preprint.

[9] G. Citti, N. Garofalo, E. Lanconelli, Harnack's inequality for sum of squares of vector fields plus a potential, Amer. J. Math. 115 (1993), 699-734. MR 94m:35069

[10] Citti, G., Lanconelli, E., Montanari, A., Smoothness of Lipschitz continuous graphs with nonvanishing Levi curvature, Acta Math. 188 (2002), 87-128.

[11] Folland, G. B., Subelliptic estimates and function spaces on nilpotent Lie groups, Ark. Mat. 13 (1975), 161-207. MR 58:13215 
[12] Franchi, B., Lu, G., Wheeden, R. L., Weighted Poincaré inequalities for Hörmander vector fields and local regularity for a class of degenerate elliptic equations, Potential Anal. 4 (1995), 361-375. MR 97e:35018

[13] Hörmander, L., Hypoelliptic second order differential equations, Acta Math. 119 (1967), 147-171. MR 36:5526

[14] Huisken, G., Klingenberg, W., Flow of real hypersurfaces by the trace of the Levi form, Math. Res. Lett. 6 (1999), 645-661. MR 2001f:53141

[15] Jerison, D., Lee, J. M., The Yamabe problem on CR manifolds, J. Differential Geom. 25 (1987), 167-197. MR 88i:58162

[16] Jerison, D., SÁnchez-Calle, A., Estimates for the heat kernel for a sum of squares of vector fields, Indiana Univ. Math. J. 35 (1986), 835-854. MR 88c:58064

[17] Kusuoka, S., Stroock, D., Long time estimates for the heat kernel associated with a uniformly subelliptic symmetric second order operator, Ann. of Math. 127 (1988), 165189. MR 89b:35022

[18] Lanconelli, E., PAscucci, A., Polidoro, S., Linear and nonlinear ultraparabolic equations of Kolmogorov type arising in diffusion theory and in finance, Nonlinear Problems in Mathematical Physics and Related Topics, II, in Honor of Professor O. A. Ladyzhenskaya, International Mathematical Series, 2, to appear.

[19] Lu, G., Existence and size estimates for the Green's functions of differential operators constructed from degenerate vector fields, Comm. Partial Differential Equations 17 (1992), 1213-1251. MR 93i:35030

[20] Montanari, A., Real hypersurfaces evolving by Levi curvature: smooth regularity of solutions to the parabolic Levi equation, Comm. Partial Differential Equations 26 (2001), 1633-1664. MR 2002i:35110

[21] Montgomery, R., A tour of subriemannian geometries, their geodesics and applications, Mathematical Surveys and Monographs 91, American Mathematical Society, Providence, RI, 2002. MR 2002m:53045

[22] Peтitot, J., Tondut, Y., Vers une neurogéométrie. Fibrations corticales, structures de contact et contours subjectifs modaux, Math. Inform. Sci. Humaines 145 (1999), 5-101. MR 2000j:92007

[23] Rothschild, L. P., Stein, E. M., Hypoelliptic differential operators and nilpotent groups, Acta Math. 137 (1976), 247-320. MR 55:9171

[24] Slodkowski, Z., Tomassini, G., Weak solutions for the Levi equation and envelope of holomorphy, J. Funct. Anal. 101 (1991), 392-407. MR 93c:32018

[25] Varopoulos, N. T., Saloff-Coste, L., Coulhon, T., Analysis and geometry on groups, Cambridge Tracts in Mathematics 100, Cambridge University Press, Cambridge, 1992. MR 95f: 43008

[26] Xu, C. J., Regularity for quasilinear second-order subelliptic equations, Comm. Pure Appl. Math. 45 (1992), 77-96. MR 93b:35042

Dipartimento di Matematica, Università degli Studi di Bologna, Piazza di Porta S. Donato 5, 40126 Bologna, Italy

E-mail address: bonfigli@dm.unibo.it

Dipartimento di Matematica, Università degli Studi di Bologna, Piazza di Porta S. Donato 5, 40126 Bologna, Italy

E-mail address: lanconel@dm.unibo.it

Dipartimento di Matematica, Università degli Studi di Bologna, Piazza di Porta S. Donato 5, 40126 Bologna, Italy

E-mail address: uguzzoni@dm.unibo.it 